\title{
Gold Nanoparticles Supported on Polyacrylamide Containing a Phosphorus Ligand as an Efficient Heterogeneous Catalyst for Three-Component Synthesis of Propargylamines in Water
}

Mohammad Gholinejad*

Fatemeh Hamed

Carmen Nájera*

Synlett 2016, 27, 1193.

For the final online and print versions, in Table 2 entries 13 and 14, the structures of the products were corrected. The furan rings were missing the double bonds. 